\title{
Multiprocessor Fixed Priority Scheduling with Limited Preemptions
}

\author{
Abhilash Thekkilakattil ${ }^{1}$, Robert I. Davis ${ }^{2}$, Radu Dobrin ${ }^{1}$, Sasikumar Punnekkat ${ }^{3}$, and Marko Bertogna ${ }^{4}$ \\ ${ }^{1}$ Mälardalen Real-Time Research Center, Mälardalen University, Sweden \\ ${ }^{2}$ Real-Time Systems Research Group, Department of Computer Science, University of York, UK \\ ${ }^{3}$ Department of Computer Science and Information Systems, Birla Institute of Technology and Science, India \\ ${ }^{4}$ University of Modena, Italy
}

\begin{abstract}
Challenges associated with allowing preemptions and migrations are compounded in multicore systems, particularly under global scheduling policies, because of the potentially high overheads. For example, multiple levels of cache greatly increase preemption and migration related overheads as well as the difficulty involved in accurately accounting for them, leading to substantially inflated worst-case execution times (WCETs). Preemption and migration related overheads can be significantly reduced, both in number and in size, by using fixed preemption points in the tasks' code; thus dividing each task into a series of non-preemptive regions (NPRs). This leads to an additional consideration in the scheduling policy. When a high priority task is released and all of the processors are executing non-preemptive regions of lower priority tasks, then there is a choice to be made in terms of how to manage the next preemption. With an eager approach the first lower priority task to reach a preemption point is preempted even if it is not the lowest priority running task. Alternatively, with a lazy approach, preemption is delayed until the lowest priority currently running task reaches its next preemption point.

In this paper, we show that under global fixed priority scheduling with eager preemptions each task suffers from at most a single priority inversion each time it resumes execution. Building on this observation, we derive a new response time based schedulability test for tasks with fixed preemption points. Experimental evaluations show that global fixed priority scheduling with eager preemptions is significantly more effective than with lazy preemption using link based scheduling in terms of task set schedulability.
\end{abstract}

\section{INTRODUCTION}

Permission to make digital or hard copies of all or part of this work for personal or classroom use is granted without fee provided that copies are not made or distributed for profit or commercial advantage and that copies bear this notice and the full citation on the first page. To copy otherwise, to republish, to post on servers or to redistribute to lists, requires prior specific permission and/or a fee.

RTNS 2015, Lile, France

Copyright 20XX ACM X-XXXXX-XX-X/XX/XX ...\$15.00.
Multicore platforms have been developed to circumvent the fact that performance gains can no longer be achieved via further increases in processor speed, due to power and thermal constraints 3. Consequently, there is a revived interest among researchers and practitioners, particularly in the field of real-time embedded systems, to leverage on this ability of multicore systems to provide higher performance. In particular, the research focus has been on the challenges associated with such a hardware-software ecosystem, for example, the associated unpredictabilities that may compromise timeliness. A compelling challenge involves minimizing preemption and migration related overheads while enabling preemptions on lower priority tasks to aid the timely completion of both higher and lower priority ones.

Preemption and migration related overheads include context switch costs, cache related preemption and migration delays (CPMD), pipeline delays, and increased bus contention costs [4] 9], all of which significantly increases pessimism in task execution times. Consequently, fully preemptive scheduling can lead to prohibitively high preemption related overheads which degrade schedulability and can potentially cause deadline failure if not accurately accounted for. Two main reasons for high preemption and migration related overheads can be identified in this case. First, there are a large number of possible preemptions and migrations (resulting from the preemptions), and second these preemptions and migrations can occur at points in the code where the associated overhead is very high, for example where there are many (useful) cache blocks. Bastoni et al. 22 showed that the overheads due to preemptions and migrations are similar.

Fully non-preemptive scheduling offers an alternative that avoids preemption related overheads, at the cost of introducing significant blocking effects. While fixed priority preemptive and non-preemptive scheduling are incomparable for single processor systems [12, and also in the case of global scheduling for multiprocessor systems [18, non-preemptive scheduling suffers from what is known as the long task problem. This is where one task has a Worst Case Execution Time (WCET) that is longer than the deadline of another task, rendering the system unschedulable on a single processor. This problem is somewhat alleviated on multiprocessor systems, since long tasks need to be executing on all processors in order to 
cause a deadline miss 18 ; however, it is still an issue.

Limited preemption scheduling has been proposed as an alternative to the fully preemptive and fully non-preemptive paradigms in order to reduce preemption and migration related overheads, while also constraining the amount of blocking and thus improving schedulability. In limited preemption scheduling, preemptions and migrations may be restricted using various mechanisms such as scheduler enforced floating non-preemptive regions, or fixed preemption points inserted into the program code. (See 10 for a survey of the various methods). Fixed preemption points, in addition to reducing the number of preemptions and migrations, have the advantage of enabling accurate estimation and accounting for preemption and migration related overheads. Further, preemption point placement can be optimized to reduce the cost of each individual preemption and the total preemption related overheads incurred within the response time of a task 4], 6], 21. Moreover, in multiprocessors, by reducing the number of preemption points, the number of potential migrations can also be reduced.

In this paper, we consider the problem of global fixed priority scheduling of real-time tasks with fixed preemption points on a multiprocessor. Here, there is an interesting choice to be made in terms of how to manage preemptions following the release of high priority tasks. Two types of approach can be identified: With an eager approach, preemption occurs as soon as possible, i.e. the first lower priority task to reach a preemption point is preempted. (Note the preempted task may not be the lowest priority running task). Alternatively, with a lazy approach, preemption is delayed until the lowest priority running task reaches a preemption point.

The first analysis for global scheduling with a lazy form of preemption was given by Block et al. in 2007 [7] called link-based scheduling. Here, when a high priority task is released, and the lowest priority running task $\tau_{i}$ is executing an NPR, then the new task is linked to the processor that is executing $\tau_{i}$ and does not preempt until that task finishes its NPR. (Full details of the method can be found in 7 and section 3.3.3. of Brandenburg's thesis [9]). Brandenburg and Anderson 8 later presented a generic schedulability analysis method that can be applied to different global scheduling algorithms and their schedulability tests.

Building on prior work on optimal fixed priority scheduling with deferred preemption on single processor systems [12, in 2013, Davis et al. 14 provided schedulability analysis for global fixed priority scheduling assuming eager preemptions, considering a simple task model with a single final non-preemptive region at the end of each task. They showed that, as in the single processor case, schedulability can be improved by carefully choosing both task priorities and the length of their final non-preemptive regions. Davis et al. [16 subsequently corrected a problem with their test. Further, they showed that the eager and lazy approaches to preemption are incomparable i.e., there are task sets that are schedulable using eager preemptions that are unschedulable using lazy preemptions and vice-versa (illustrated by worked examples in the appendix of [16]). Later in 2013, Marinho et al. 20. generalized the work of Davis et al. 14. to cover task sets with fixed preemption points and hence multiple non-preemptive regions. Marinho et al. considered both eager and lazy forms of preemption under global fixed priority scheduling; however, they only gave analysis of the blocking effects, and thus stopped short of providing a full schedulability analysis for eager preemptions. Recently, related work has also been published on global EDF with limited preemption 24, 11.

In this paper, we address the problem of Global Limited Preemption Fixed Priority Scheduling (G-LP-FPS) for tasks with fixed preemption points, and consider how the choice of either an eager or a lazy approach to preemption affects schedulability. The main contributions of the paper are as follows:

1. The introduction of a response time based schedulability test for real-time task sets with fixed preemption points scheduled using G-LP-FPS with eager preemptions. To the best of our knowledge, this is the first such test in the context of G-LP-FPS with fixed preemption points.

2. An evaluation of G-LP-FPS with eager preemptions using the new schedulability test, against G-LP-FPS with lazy preemptions (using link-based scheduling 7]); evaluations shows the that G-LP-FPS with eager preemptions outperforms link-based scheduling for a wide range of settings.

The remainder of the paper contains the system model in Section 2 background in Section 3 , the main contributions in Section 4, followed by the evaluations in Section 5 before concluding in Section 6 .

\section{SYSTEM MODEL}

In this section, we present the system model, terminology and definitions assumed in the rest of the paper.

\subsection{Task Model}

We consider a set of $n$ sporadic real-time tasks $\Gamma=$ $\left\{\tau_{1}, \tau_{2}, \ldots \tau_{n}\right\}$ scheduled on $m$ identical processors. The tasks in $\Gamma$ are indexed according to their decreasing unique priorities i.e., $\tau_{1}$ has the highest priority and $\tau_{n}$ the lowest, and each task generates an infinite number of jobs. Let $h p(i)$ denote the subset of tasks with priorities greater than $\tau_{i}$ and $l p(i)$ denote the subset of tasks with priorities lower than $\tau_{i}$. Each task $\tau_{i}$ is characterized by a minimum inter-arrival time $T_{i}$, and a relative deadline $D_{i} \leq T_{i}$, and is assumed to contain $q_{i} \geq 0$ optimal preemption points 21. The start of the task execution is referred to as the $0^{t n}$ preemption point while the end of the task execution is referred to as the $q_{i}+1^{\text {th }}$ point; however, preemption of the task is of course not possible at these points. Let $b_{i, j}$, $j=1 \ldots q_{i}+1$ denote the worst case execution time of task $\tau_{i}$ between its $j-1^{t h}$ and $j^{t h}$ preemption points (The calculation of the WCET between preemption points is an important problem on multicores; but this is not our focus. Instead we focus on the related problem of the prohibitive increase of WCETs due to preemption related overheads). We use the notation $b_{i, j}$ to also refer to the corresponding Non-Preemptive Region (NPR). In any time interval of length $t$, each task $\tau_{i}$ can be preempted by a higher priority task at most $h_{i}$ times where $h_{i}=\sum_{\forall \tau_{j} \in h p(i)}\left\lceil\frac{t}{T_{j}}\right\rceil$. Therefore, an upper-bound on the number of preemptions $p_{i}$ of task $\tau_{i}$ in an interval of length $t$ is given by:

$$
p_{i}=\min \left(q_{i}, h_{i}\right)
$$




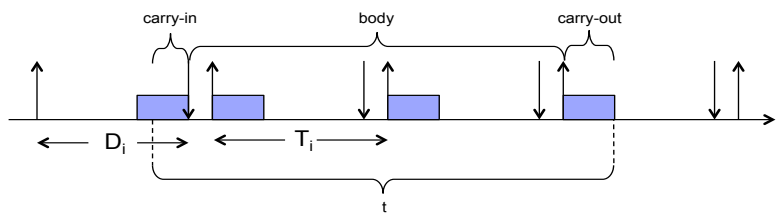

Figure 1: Task with carry-in in an interval of length $t$.

The Worst Case Execution Time (WCET) of each task $\tau_{i}$ can be calculated as $C_{i}=\sum_{j=1}^{q_{i}+1} b_{i, j}$. Note that the preemption related overheads can be integrated into the above equation, since we are interested in comparing lazy and eager preemption mechanisms and these overheads are the same in each case, we omit their specific consideration. Similar to Davis et al. [16], we also define $C_{i}^{*}$ for each $\tau_{i}$, where $C_{i}^{*}=C_{i}-b_{i, q_{i+1}}+1$. This is because, when using $C_{i}^{*}$ instead of $C_{i}$, the resulting response time implies the completion of $C_{i}-b_{i, q_{i+1}}+1$ units of execution and hence gives the start time of the final NPR (the +1 ensures start of the final NPR).

\subsection{Definitions}

According to our model every task is composed of a set of non-preemptive regions with specified lengths. This allows us to define the preemptability of a task.

Definition 2.1. Any task $\tau_{i} \in \Gamma$ is defined to be preemptable at time instant $t$ if and only if time instant $t$ corresponds to a preemption point $k, 1 \leq k<q_{i}$, in the progress of its execution.

An unfinished task is defined to be ready if it is not currently executing (and hence it is assumed to be in the ready queue). Non-preemptive regions within the lower priority tasks can cause priority inversions on higher priority tasks. We enumerate three conditions that are necessary for a single priority inversion to occur due to an NPR of a lower priority task under limited preemption scheduling.

DeFINITION 2.2. A priority inversion occurs on an arbitrary task $\tau_{i}$ when the following conditions hold.

C1: The scheduler is invoked by $\tau_{i}$, and $\tau_{i}$ is ready.

C2: At least one processor is executing a lower priority task.

C3: All the lower priority jobs are not preemptable.

We differentiate the following types of interference for any task $\tau_{i}$.

DEFINITION 2.3. The higher priority interference on a task $\tau_{i}$ is defined as the cumulative executions of all tasks having a higher priority than $\tau_{i}$ that prevent $\tau_{i}$ from executing on the processor.

DEFINITION 2.4. The lower priority interference on a task $\tau_{i}$ is defined as the cumulative executions of all tasks having a lower priority than $\tau_{i}$ that prevent $\tau_{i}$ from executing on a processor.

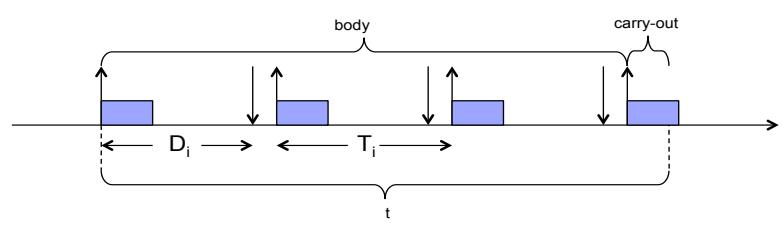

Figure 2: Task without carry-in in an interval of length $t$.

We use $I_{i}\left(t, \tau_{j}\right)$ to denote the interference on $\tau_{i}$ from a higher priority task $\tau_{j}$ in the time interval of length $t$ and $I_{i}(t)$ to denote the total higher priority interference. Finally, we note that the final NPR of the jobs of any $\tau_{i}$ delays the start of higher priority tasks released during its execution, which may in turn interfere with the next release of $\tau_{i}$. This interference pushed through by any job of a task on to its next job is defined as push though blocking (see section 1.4 of 13. for an example).

\section{BACKGROUND}

In this section, we review the state-of-the-art schedulability analysis for global fixed priority scheduling under the preemptive and limited preemption paradigms.

\subsection{Schedulability Analysis for Global Preemptive FPS}

Bertogna, Cirinei and Lipari 5 derived an upper-bound on the interference generated by any task $\tau_{j}$ on a lower priority task $\tau_{i}$ over an interval of length $t$, under Global Preemptive Fixed Priority Scheduling (G-P-FPS) and used this to derive a response time analysis. Even though this analysis has subsequently been improved upon by others, we recall this specific result because of its seminal nature. The upper-bound on the interference generated by any task $\tau_{j}$ on a lower priority task $\tau_{i}$ over an interval of length $t$ is given by:

$$
I_{i}\left(t, \tau_{j}\right)=\min \left(I_{i}^{\prime}\left(t, \tau_{j}\right), t-C_{i}+1\right)
$$

where,

$$
I_{i}^{\prime}\left(t, \tau_{j}\right)=N_{j}(t) C_{j}+\min \left(C_{j}, t+D_{j}-C_{j}-N_{j}(t) T_{j}\right)
$$

In the above equation, $N_{j}(t)=\left\lfloor\frac{t+D_{j}-C_{j}}{T_{j}}\right\rfloor$. Consequently, any task $\tau_{i}$ is schedulable if,

$$
R_{i}=C_{i}+\left\lfloor\frac{1}{m} \sum_{\tau_{j} \in h p(i)} I_{i}\left(R_{i}, \tau_{j}\right)\right\rfloor \leq D_{i}
$$

Baruah 1] observed that the pessimism in the interference calculation can be attenuated by considering a larger time interval. Baruah also observed that at the time instant earlier than the release of $\tau_{i}$ where at least one processor is idle, the number of jobs that have carry-in workload is at most $m-1$ (see figure 1 and 2 for an illustration of workloads with and without carry-in)). The response time analysis of Bertogna, Cirinei and Lipari, was improved by Guan et al. 17. by instantiating these observations in the context of G-P-FPS to limit the carry-in interference. Later Sun et al. 23. identified and fixed some anomalies in the test for the arbitrary deadline case. In order to 
determine the schedulability of tasks with fixed preemption points, similar to [16], we define:

$$
I_{i}\left(t, \tau_{j}\right)=\min \left(I_{i}^{\prime}\left(t, \tau_{j}\right), t-C_{i}^{*}+1\right)
$$

where $I_{i}^{\prime}\left(t, \tau_{j}\right)$ is given by $(3)$.

\subsection{Schedulability analysis with lazy preemption}

In this section, we present schedulability analysis for Global Limited Preemption Fixed Priority Scheduling (G-LP-FPS) with lazy preemptions using link-based scheduling as proposed by Block et al. 7. First we present an example illustrating lazy preemptions.

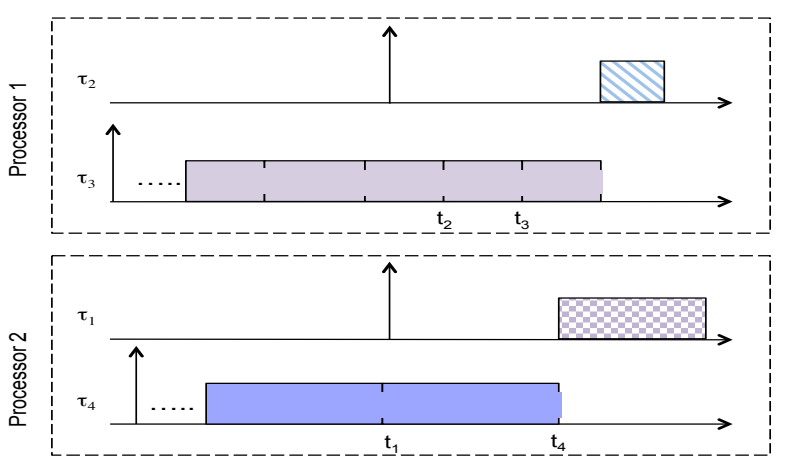

Figure 3: Example schedule illustrating lazy preemption.

Example 3.1. Consider the scenario in Figure 3 where 4 tasks $\tau_{1}, \tau_{2}, \tau_{3}$ and $\tau_{4}$ (in decreasing priority order) are executing on two processors. Assume that tasks $\tau_{3}$ and $\tau_{4}$ are executing on the processor at time instant $t_{1}$. Suppose that $\tau_{1}$ and $\tau_{2}$ are released together at time instant $t_{1}$, and the scheduling policy uses lazy preemptions. In this case, $\tau_{1}$ starts executing only at time $t_{4}$ even though a lower priority task $\left(\tau_{3}\right)$ was available to preempt earlier (at $t_{2}$ ). Moreover, task $\tau_{2}$ is blocked 3 times by $\tau_{3}$ since $\tau_{4}$ has to be preempted first. At time $t_{4}$, when the scheduler is invoked, $\tau_{4}$ is preempted by $\tau_{1}$. However, since task $\tau_{3}$ is not preemptible $\tau_{2}$ still cannot start executing. The total number of such priority inversions could be arbitrarily large if $\tau_{4}$ has a large non-preemptive region.

Davis et al. 16 14 enumerated a number of interesting observations regarding the blocking introduced by the priority inversions that occur due to non-preemptive execution of lower priority tasks: 1) The number of priority inversions is not limited to the number of processors $m$, as is the case with Global Non Preemptive Fixed Priority Scheduling (G-NP-FPS) 2) More than one job of the same lower priority tasks can cause priority inversion on a higher priority task 3) More than one non-preemptive region of the same job of each lower priority task can cause priority inversions.

Link based scheduling: Many of these challenges are addressed by link-based scheduling developed by Block et al. 7. in the context of resource sharing, which is equally applicable to the problem of limited preemption scheduling with NPRs. Link-based scheduling implements a form of lazy preemptions whereby a newly released high priority task is linked to the processor executing the lowest priority task. Even though link-based scheduling emulates floating NPR scheduling, it can be used in the context of fixed preemption points scheduling by considering the floating NPRs to be fixed.

Link based scheduling analysis: The analysis for link-based scheduling presented by Block et al. 7 and Brandenburg and Anderson [8] provides a simple generic means of accounting for blocking due to NPRs. It uses an inflation based method, in which the WCETs of higher priority tasks are inflated to account for blocking from lower priority tasks. Specifically, $\forall \tau_{i} \in \Gamma$, the associated WCET is inflated as follows:

$$
C_{i}=C_{i}+\max _{\tau_{j} \in l p(i)} b_{j, k}, \quad k=1, \ldots, q_{i}^{1}
$$

Brandenburg and Anderson 8] proved that the resulting taskset $\Gamma^{\prime}$ obtained by inflating the WCETs of all the tasks in $\Gamma$ with the maximum blocking that they can suffer, is a safe hard real-time approximation 9 . This means that if there is a deadline miss with link-based scheduling and NPRs, then there is also guaranteed to be a deadline miss in $\Gamma^{\prime}$ under fully preemptive scheduling. Consequently, a fully preemptive schedulability analysis such as that given in 17 can be applied to determine schedulability.

\section{SCHEDULABILITY ANALYSIS WITH EAGER PREEMPTIONS}

In this section, we present the main contributions of this paper. Specifically, we examine G-LP-FPS with eager preemptions in which the highest priority task is allowed to preempt the first lower priority job that becomes preemptable. We show that the number of priority inversions on any task $\tau_{i}$ is upper-bounded by the associated upper-bound on the number of preemptions $p_{i}$ (defined in (1)). Building on this observation, we derive a response time analysis based test for schedulability under G-LP-FPS with eager preemptions.
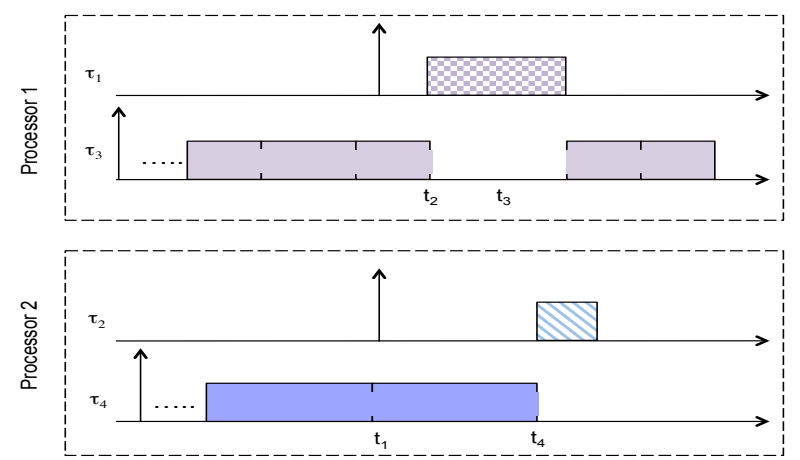

Figure 4: Example schedule illustrating eager preemptions.

In the following we illustrate how G-LP-FPS with eager preemptions schedules the taskset in Example 3.1

Example 4.1. Consider the same scenario presented in Figure 3 where $\tau_{1}$ and $\tau_{2}$ are released at time instant $t_{1}$. As with eager preemptions, illustrated in Figure 4. tasks $\tau_{1}$ and $\tau_{2}$ are blocked at time instant $t_{1}$. When $\tau_{3}$ finishes executing its non-preemptive region, $\tau_{1}$ is scheduled on processor 1. 
When $\tau_{4}$ finishes executing its non-preemptive region, $\tau_{2}$ can start its execution.

Recall that Example 3.1 showed that with lazy preemption every time a task is inserted into the ready queue after it is released, it may suffer from more than one priority inversion. Eager preemptions enable the medium priority task $\tau_{2}$ to be allocated to the processor earlier than is the case with lazy preemption, as illustrated in Example 4.1. We now derive a bound on the number of priority inversions with eager preemptions.

LEMMA 4.1. The number of priority inversions on any task $\tau_{i}$ that is inserted into the ready queue under $G-L P$ FPS with eager preemptions is at most 1 before it can again start executing.

Proof. Consider the time instant when $\tau_{i}$ is inserted into the ready queue and all the processors are executing the NPRs of lower priority tasks. Let $\tau_{k}$ be the lower priority executing task having the longest duration to the next preemption point from among the executing tasks. When one of the lower priority tasks reaches its preemption point, the scheduler is invoked and the next highest priority task from the ready queue is scheduled. When the next preemption point of $\tau_{k}$ is reached, either $\tau_{i}$ will be (or would have been) scheduled or all processors will be executing tasks having priority higher than $\tau_{i}$. No new non-preemptive regions of lower priority tasks can start executing since there are higher priority tasks waiting in the ready queue. Therefore, the number of priority inversions on $\tau_{i}$ is at most 1 whenever it is inserted into the ready queue.

We can upper-bound the number of priority inversions on any task using knowledge of the number of preemption points and an upper-bound on the number of preemptions that it can suffer.

COROllary 4.1. The number of priority inversions on any task $\tau_{i}$ under G-LP-FPS with eager preemptions in any time interval of length $t$ is at most $p_{i}$, where $p_{i}$ is defined in (1).

Proof. According to Lemma 4.1 $\tau_{i}$ suffers from at most 1 priority inversion every time it is inserted into the ready queue. Recall that each task $\tau_{i}$ can be preempted at most $p_{i}$ times during any interval of length $t$. Therefore, $\tau_{i}$ can be inserted into the ready queue most $p_{i}$ times after it starts, i.e., whenever it is preempted.

Task execution dynamics on multiprocessors: Phillips et al. 22 noted that, under preemptive scheduling, in any time interval of length $t$ between the release time and deadline of a task $\tau_{i}$, whenever $\tau_{i}$ is not executing, all processors are executing higher priority jobs. On the other hand, when tasks are composed of non-preemptive regions, Phillips et al. 's observation needs to be modified as follows: In any time interval of length $t$, whenever a task $\tau_{i}$ is ready and is not executing, the processor is executing higher priority tasks, or by lower priority NPRs of tasks blocking $\tau_{i}$. This observation can be applied to obtain the time at which any non-preemptive region of task $\tau_{i}$ can start executing, which in turn gives us the corresponding response time.
In the following two lemmas, we present an upper-bound on the lower priority interference on 1) the first NPR of any task $\tau_{i}$ and 2) any other NPR of $\tau_{i}$.

The lower priority interference on the first NPR of $\tau_{i}$ needs to account for the push through blocking which is the interference pushed through by the final NPR of the previous job of $\tau_{i}$. However, as noted by Davis et al. [16, when calculating the higher priority interference using Bertogna et al. 's 5 method, since the higher priority tasks are assumed to be executing as late as possible, the effects of push through blocking is already accounted for. We therefore, obtain the following upper-bound on the blocking on the first NPR of any $\tau_{i}$.

LEMMA 4.2. The lower priority interference without accounting for the push through blocking on the first NPR $b_{i, 1}$ of a task $\tau_{i}$ over all $m$ processors under G-LP-FPS with eager preemptions is upper-bounded by

$$
\Delta_{i}^{m}=\sum \max _{\tau_{j} \in l p(i)}^{m}\left(\max _{1 \leq k \leq q_{j+1}} b_{j, k}\right)
$$

where, the $\sum \max _{\tau_{j} \in l p(i)}^{m}$ term denotes the sum of the $m$ largest values among the NPR's of all $\tau_{j} \in l p(i)$.

Proof. This follows from the fact that at most $m$ tasks can be executing at any given time instant, and eager preemptions guarantee that the first preemptable lower priority task is preempted by a higher priority task.

In the worst case, when $\tau_{i}$ is released, all the $m$ processors have just started executing the $m$ largest lower priority NPRs. Consequently, with eager preemptions $\tau_{i}$ needs to wait until these $m$ largest NPRs of lower priority tasks complete their execution before all the processors are busy executing either tasks having higher priority than $\tau_{i}$ (in which case, there is no more priority inversion) or $\tau_{i}$ itself.

Lemma 4.3. The lower priority interference on the $p^{\text {th }}$ non-preemptive region $b_{i, p}$ of any task $\tau_{i}$ over all $m$ processors under G-LP-FPS with eager preemptions is upper-bounded by

$$
\Delta_{i}^{m-1}=\sum \max _{\tau_{j} \in l p(i)}^{m-1}\left(\max _{1 \leq k \leq q_{j}+1} b_{j, k}\right)
$$

where, $2 \leq p \leq q_{i}+1$ and $\sum \max _{\tau_{j} \in l p(i)}^{m-1}$ denotes the sum of the $m-1$ largest values among all $\tau_{j} \in l p(i)$.

Proof. When $\tau_{i}$ is executing, then at most $(m-1)$ processors are executing lower priority tasks. Suppose that there exists a time instant between the start time and finish time of $\tau_{i}$ when all the processors are executing lower priority tasks. Let $t$ denote the earliest such time instant. This means that at time instant $t$, the scheduler scheduled a new low priority job (i.e., an $m^{\text {th }}$ lower priority task) even though $\tau_{i}$ was waiting in the ready queue or was executing. We get a contradiction because of our assumption of an global fixed priority based scheduler.

LEMMA 4.4. An arbitrary task $\tau_{i}$ can start executing its final NPR $q_{i}+1$, in any time interval of length $t$ under $G-L P$ FPS with eager preemptions if $\tau_{i}$ is ready at the beginning 
of the interval and,

$$
C_{i}^{*}+\left\lfloor\frac{1}{m}\left(\Delta_{i}^{m}+p_{i} \times \Delta_{i}^{m-1}+\sum_{\tau_{j} \in h p(i)} I_{i}\left(t, \tau_{j}\right)\right)\right\rfloor \leq t
$$

where, $p_{i}$ is given by (1), $\Delta_{i}^{m}$ is given by (6), $\Delta_{i}^{m-1}$ is given by (7) and $I_{i}\left(t, \tau_{j}\right)$ is given by (5).

Proof. Recall that $\Delta_{i}^{m}$ denotes the sum of the largest lower priority NPRs that can block $\tau_{i}$ over all $m$ processors and $I_{i}\left(t, \tau_{j}\right)$ gives the worst case interference in the interval $t$. We know from Lemma 4.1 that the each NPR of $\tau_{i}$ can be blocked at most once. Moreover, we know from lemmas 4.2 and 4.3 that the first NPR of $\tau_{i}$ can be blocked by at most $\Delta_{i}^{m}$ and that each of the remaining NPRs of $\tau_{i}$ can be blocked at most $\Delta_{i}^{m-1}$ over all the $m$ processors. Moreover, the number of preemptions on $\tau_{i}$ is upper-bounded by $p_{i}$. In the worst case, whenever $\tau_{i}$ resumes its execution after a preemption, it is blocked by lower priority NPRs. Therefore the upper-bound on the blocking experienced by $\tau_{i}$ is given by $\Delta_{i}^{m}+p_{i} \times \Delta_{i}^{m-1}$.

Proof follows from the fact that $\tau_{i}$ has completed $C_{i}^{*}=$ $C_{i}-b_{i, q_{i}+1}+1$ units of execution, after incurring the worst case higher and lower priority interference, implying that the final NPR has already started its execution.

In the following, we present a schedulability test by observing that any task $\tau_{i}$ can be blocked only when it is preempted, and the number of preemptions on $\tau_{i}$ is at most $p_{i}$.

THEOREM 4.1. If for any task $\tau_{i}$, suppose $t^{\prime}$ denotes the smallest $t$ for which equation (8) is satisfied, the response time of $\tau_{i}$ under G-LP-FPS with eager preemptions is given by,

$$
R_{i}=C_{i}+\left\lfloor\frac{1}{m}\left(\Delta_{i}^{m}+p_{i} \times \Delta_{i}^{m-1}+\sum_{\tau_{j} \in h p(i)} I_{i}\left(t^{\prime}, \tau_{j}\right)\right)\right\rfloor
$$

where $\Delta_{i}^{m}$ and $\Delta_{i}^{m-1}$ are defined in (6) and (7) respectively.

Proof. The proof follows from the fact that, at $t^{\prime}$, the final NPR of $\tau_{i}$ has started its execution.

The smallest $t$ that satisfies (8) can be obtained by first setting $t=C_{i}^{*}$ and performing a fixed point iteration on (8) until the condition is satisfied or until a value greater than $D_{i}-b_{i, q+1}+1$ is obtained, in which case the task is unschedulable.

The test presented above is, however, pessimistic since it assumes that all the higher priority tasks have carry-in jobs that interfere with $\tau_{i}$. In the following, we build on Baruah's observations [1, and identify a critical scenario that gives the worst case behavior under limited-preemption scheduling while limiting the number of tasks with carry-in jobs. Baruah [1] observed that the worst case scenario that leads to a deadline miss on any job of $\tau_{i}$, under preemptive scheduling, is such that there exists a busy interval prior to the release of $\tau_{i}$ where all the processors are executing higher priority jobs and extends to the time instant when the job of $\tau_{i}$ can start executing. The start time of such a busy period is assumed to be the earliest time before the release of $\tau_{i}$ such that at least one processor is idle and no processors are idle between the start of the busy period and

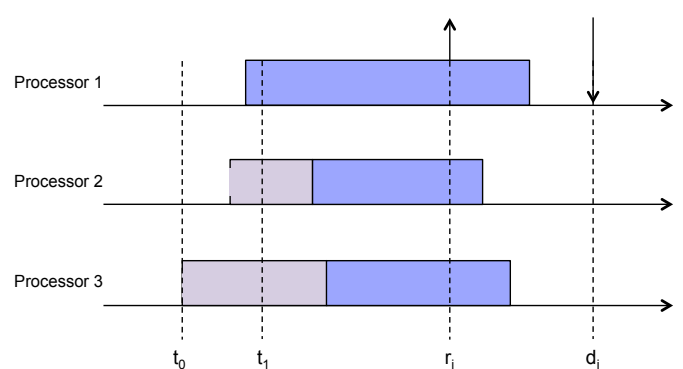

Low priority task executions

High priority task executions

Figure 5: An illustration of the critical scenario.

the release of $\tau_{i}$. We build on this observation by identifying that, in the case of limited-preemption scheduling, the NPRs of some lower priority jobs could influence the busy period and consider the following scenario.

Critical Scenario: Consider an arbitrary job $J_{i}$ of a task $\tau_{i}$ released at time instant $r_{i}$ that has started its execution, such that all jobs of all tasks released prior to $r_{i}$ are schedulable. We consider the earliest time instant $t_{1}$ before the release time $r_{i}$ at which a higher priority task is released, and is blocked by NPRs of some tasks having a lower priority than $\tau_{i}$, as illustrated in Figure 5. If there are no higher priority job releases prior to $r_{i}$, we set $t_{1}=r_{i}$ (i.e., assume $J_{i}$ is the task that is blocked). Let the number of processors executing lower priority NPRs at time instant $t_{1}$ be $x, 1 \leq x \leq m$. Let $t_{0}$ denote the earliest among the start times of these $x$ lower priority NPRs that block the higher priority task released at $t_{1}$. If there is no such time instant, we set $t_{0}$ to be the earliest time instant before $r_{i}$ at which at least one processor is idle and no processor is idle in $\left(t_{0}, r_{i}\right)$ (there is always such an instant e.g., at the start of the schedule). Since we consider a sporadic task system, there is always a possibility of lower priority tasks being released, and being executed, at $t_{0}$ that may potentially block the execution of $\tau_{i}$.

OBSERVATION 4.1. At most $m-1$ higher priority tasks are active at time instant $t_{0}$.

According to our identified critical scenario, at least one processor is starting to execute a lower priority task at time instant $t_{0}$. This means that except for the higher priority tasks currently executing on at most $m-1$ processors no other higher tasks are active. If more than $m-1$ higher priority tasks were active, lower priority tasks could not have started executing at $t_{0}$.

The above observation allows us to limit the number of carry-in tasks to at most $m-1$, rather than assuming that all the tasks have carry-in executions. Consequently, we can exploit the recent advances 17. in efficiently accounting for the carry-in interference to determine the schedulability of real-time tasks under G-LP-FPS with eager preemptions. The workload generated by any higher priority task $\tau_{j}$ having carry-in interference in any time interval of length $t$ is given as follows 17. (see Figure 1 for an illustration):

$$
W_{j}^{C I}(t)=C_{j}+\left\lfloor\frac{\max \left(\left(t-C_{j}\right), 0\right)}{T_{j}}\right\rfloor \times C_{j}+
$$




$$
\min \left(\left[\left[t-C_{j}\right]_{0} \bmod T_{j}-\left(T_{j}-R_{j}\right)\right]_{0}, C_{j}\right)
$$

In the above equations, $[A]_{B}$ means $\max (A, B)$. On the other hand, the workload generated by any higher priority task $\tau_{j}$ that does not carry-in interference in any time interval of length $t$ is given as follows 17. (see Figure 2 for an illustration):

$$
W_{j}^{N C}(t)=\left\lfloor\frac{t}{T_{j}}\right\rfloor \times C_{j}+\min \left(t \bmod T_{j}, C_{j}\right)
$$

Therefore, in any time interval of length $t$ and for any $\tau_{j}$, we can calculate the upper-bound on the associated carry-in as follows 17: $W_{j}^{\text {diff }}(t)=W_{j}^{C I}(t)-W_{j}^{N C}(t)$. A conservative upper-bound on the amount of carry-in by higher priority tasks that interferes with any task $\tau_{i}$ can be obtained from the $(m-1)$ largest $W_{j}^{\text {diff }} \mathrm{s}$ :

$$
I_{i}^{C I}(t)=\sum \max _{\tau_{j} \in h p(i)}^{m-1} W_{j}^{\text {diff }}(t)
$$

Similarly, a conservative upper-bound on the interference generated by higher priority tasks on $\tau_{i}$, that do not carry$i n$, in any time interval of length $t$ is given by,

$$
I_{i}^{N C}(t)=\sum_{\tau_{j} \in h p(i)} W_{j}^{N C}(t)
$$

Therefore, the total higher priority interference on any task $\tau_{i}$ in any time interval of length $t$ can be calculated using:

$$
I_{i}(t)=I_{i}^{C I}(t)+I_{i}^{N C}(t)
$$

However, when restricting the number of carry-in jobs to at most $m-1$, the tasks are not assumed to be executing as late as possible. Consequently, as noted by Davis et al. [16, the effects of push through blocking is not automatically accounted for. In the following we show that, for any $\tau_{i}$, only at most one final NPR of a previous job of $\tau_{i}$ can contribute to the push through blocking and the push through blocking can affect only its first NPR.

We consider the critical scenario described above and investigate the push through blocking on the job $J_{i}$ of $\tau_{i}$.

LEMMA 4.5. The push through blocking on $\tau_{i}$ comes from at most the final NPR of the previous job of $\tau_{i}$, under $G$ $L P$-FPS with eager preemptions.

Proof. According to the critical scenario, all jobs released prior to the release time of the job $J_{i}$ of $\tau_{i}$ are schedulable, including any previous jobs of $\tau_{i}$. This means that the final NPR of the previous job of $J \tau_{i}$ had started executing. Therefore, the push through blocking from its previous NPR must have ended. Therefore, the blocking on $J_{i}$ comes from only at most the previous job of $\tau_{i}$ and its final NPR.

Lemma 4.6. Only the first NPR of $\tau_{i}$ can be affected by the push through blocking, under G-LP-FPS with eager preemptions.

Proof. Follows from the fact that the push through blocking needs to end before the first NPR of $\tau_{i}$ can start executing, and hence does not affect any subsequent NPRs.

Lemmas 4.5 and 4.6 allows us to upper-bound the lowerpriority interference, including any push through blocking, on the first NPR of any $\tau_{i}$ (the blocking on other NPRs of $\tau_{i}$ remains the same as in (7).
LEMma 4.7. The lower priority interference on the first $N P R b_{i, 1}$ of a task $\tau_{i}$ over all the $m$ processors under $G-L P$ FPS with eager preemptions can be upper-bounded by

$$
\Delta_{i}^{m}=\sum \max _{\tau_{j} \in l p(i)}\left(\beta_{i}\right)
$$

where, set $\beta_{i}=\left\{\tau_{j} \in l p(i), \max _{1 \leq k \leq q_{j+1}} b_{j, k}\right\} \cup\left\{b_{i, q_{i}+1}\right\}$, and $\sum \max _{\tau_{j} \in l p(i)}^{m}$ term denotes the sum of the $m$ largest values.

Proof. At the release time of $\tau_{i}$, when calculating blocking, we have two cases:

1. All $m$ processors are executing lower priority NPRs.

In this case, there is no push through blocking since it must have ended for all the processors to be executing lower priority NPRs. The worst case blocking in this case is given by the $m$ largest lower priority NPRs.

2. At least one processor is executing a higher priority task.

In this case, the higher priority tasks may bring in push through blocking. Therefore, the worst case blocking on $\tau_{i}$ happens when $m-1$ processors are executing the $m-1$ largest NPRs and the $m^{t h}$ processor is executing the highest priority job that brings in a push through blocking. The push through blocking is at most $b_{i, q_{i}+1}$ according to lemmas 4.5 and 4.6

Therefore, the worst case blocking on the first NPR of any $\tau_{i}$ is obtained by taking the maximum of the two cases described above.

The Lemma 4.4 can be modified as follows to compute the start time of the final NPR.

LEMma 4.8. An arbitrary task $\tau_{i}$ can start executing its final $N P R q_{i}+1$, in any time interval of length $t$ under $G-L P$ FPS with eager preemptions if $\tau_{i}$ is ready at the beginning of the interval and,

$$
C_{i}^{*}+\left\lfloor\frac{1}{m}\left(\Delta_{i}^{m}+p_{i} \times \Delta_{i}^{m-1}+I_{i}(t)\right)\right\rfloor \leq t
$$

where, $p_{i}$ is given by (1), $\Delta_{i}^{m}$ is given by (12), $\Delta_{i}^{m-1}$ is given by (7) and $I_{i}(t)$ is given by (11).

Consequently, the response time of any $\tau_{i} \in \Gamma$ can be calculated by modifying Theorem 4.1, as follows:

TheOREM 4.2. For any task $\tau_{i}$, suppose $t^{\prime}$ denotes the smallest $t$ for which equation (13) is satisfied, the response time of $\tau_{i}$ under G-LP-FPS with eager preemptions is given by,

$$
R_{i}=C_{i}+\left\lfloor\frac{1}{m}\left(\Delta_{i}^{m}+p_{i} \times \Delta_{i}^{m-1}+\sum_{\tau_{j} \in h p(i)} I_{i}\left(t^{\prime}\right)\right)\right\rfloor
$$

where $\Delta_{i}^{m}$ and $\Delta_{i}^{m-1}$ are defined in (12) and (7) respectively and $I_{i}\left(t^{\prime}\right)$ is defined in (11).

While it may seem that accounting for worst case lower priority interference per preemption on each task is pessimistic, it is sufficient to guarantee the absence of scheduling anomalies. For example, it may happen that some higher priority tasks execute for less than their worst case execution time and a lower priority task starts executing a large NPR in the resulting slack. 


\section{EVALUATIONS}

In this section, we report the results of an experimental evaluation of the performance of G-LP-FPS with eager and lazy preemptions using weighted schedulability 2. For this purpose, we used the test derived in this paper for the eager preemption approach (EPA). The schedulability under lazy preemption approach (LPA) was determined for link-based scheduling [7, using the inflation based approach 8 in conjunction with the schedulability test for G-P-FPS given by Guan et al. 17]. We calculated the weighted schedulability variations with respect to 1) number of tasks per taskset 2) the NPR lengths and 3) number of processors. Specifically, we varied:

1. the number of tasks keeping the number of processors and NPR lengths constant

2. the NPR lengths keeping the number of processors and the number of tasks constant for tasks with 1) long NPRs relative to their WCET and 2) short NPRs relative to their WCET

3. the number of processors keeping the number of tasks and NPR lengths constant

A higher weighted schedulability implies a better scheduling algorithm since the schedulability is weighted against taskset utilizations. For reference, we also included the weighted schedulabilities under G-P-FPS assuming no overheads and G-NP-FPS; the performance of G-P-FPS will significantly decrease relative to the other algorithms when overheads are included since the pessimism associated with overhead accounting is much higher. Detailed evaluation via analysis including overheads and measurements from a real implementation are future work. Moreover, the behavior of G-P-FPS can be obtained using G-LP-FPS by allowing preemptions after every unit of execution, with ties broken using task priority, and that of G-NP-FPS can be obtained by having no preemption points.

\subsection{Experimental Setup}

We used the UUnifast-Discard algorithm proposed by Davis and Burns [15] to generate task utilizations. The minimum separation times (periods) were uniformly generated between 50 and 500 (note that the time period ranges are changeable). Deadlines were set equal to periods (implicit deadlines), although the schedulability tests also apply to constrained-deadline tasksets. The largest NPR values for each $\tau_{i} \in \Gamma$ were set as a percentage of its WCET denoted by $\mathcal{P}$ (ceiling function was applied to get integer values). We assumed that all NPRs of $\tau_{i}$ have the same length equal to the largest value, except for the first that can be smaller (depending on the WCET). Note that prior work [14] shows that larger final NPRs give improved schedulability. The utilization of the tasksets ranged from a minimum of $U_{t o t}^{m i n}$ to a maximum of $U_{\text {tot }}^{\max }=m$, where $m$ is the number of processors. In the experiments, we set $U_{\text {tot }}^{\min }=2.4$ since we are more interested in scheduling tasks with larger utilizations to effectively use the platform. We assumed Deadline Monotonic Priority ordering (DMPO) 19]; although better priority assignment algorithms exists for G-P-FPS DMPO serves our purpose of comparison since we use the same priority assignment for all the considered scheduling algorithms. The test in 17. was adopted as reference for G-P-FPS, and for G-NP-FPS, we used our test after setting the largest NPR length equal to the task computation times.

\subsection{Experimental Results}

In the first experiment, we examined the performance of G-LP-FPS for varying numbers of tasks for $m=4$ processors and $\mathcal{P}=5 \%$. The results of the experiments are illustrated in Figure 6

The experiment indicates that for high utilization tasksets with large numbers of tasks, G-LP-FPS with eager preemptions performs better than with lazy preemptions. This is due to the inherent pessimism in the inflation based technique, which is amplified for large tasksets and large utilizations. We also observe that the performance of G-NP-FPS improved significantly with an increasing number of tasks. The main reason is that when the number of tasks is large, the individual task utilizations are very small, hence most tasks have relatively small computation times in relation to their deadlines and so are more amenable to G-NP-FPS [18. By contrast, the presence of preemption points introduces additional blocking (from lower priority interference occurring after the start of the execution) leading to reduced schedulability. Evaluations for larger numbers of processors (specifically $m=6$ and $m=8$ ), varying the number of tasks showed similar behavior 1

We also investigated the consequences of changing NPR lengths, and consequently the number of preemption points, on schedulability. Increasing NPR lengths increases pessimism in link-based scheduling because of the inflation of WCETs. However, it can have beneficial effects in our test for G-LP-FPS under eager preemption. We varied the size of NPRs as a percentage of the corresponding WCETs, between $5 \%$ and $100 \%$ (approximated using a ceiling function). The results are presented in Figure 7

As can be seen the schedulability varies in a saw-tooth manner until the NPR lengths reach $50 \%$ (i.e., the number of preemption points reduces to 1 ). Once past $50 \%$, the schedulability continues to decrease until the tasks are almost fully non-preemptive, after which the schedulability starts to increase again. This is because, once the NPR lengths go past $50 \%$ of WCET, there is no further reduction in the number of preemption points; however, the length of the largest NPRs continues to increase, consequently increasing blocking on higher priority tasks (that may have a single preemption point). Finally, when the tasks become fully non-preemptive, schedulability increases since the lower priority interference decreases due to the reduction in the number of preemption points from one to zero.

The schedulability varies in a saw-tooth fashion between $5 \%$ and $50 \%$ demonstrating the futility of increasing the largest NPR lengths without reducing the number of preemption points. For example, as we increase the largest NPR lengths from $25 \%$ to $30 \%$, there is no further decrease in the number of preemption points (which is 3 for both cases). On the other hand, the NPR lengths increase leading to increased lower priority interference that occurs after the start of the task executions, reducing

${ }^{1}$ see additional graphs available online at http://www.idt. mdh.se/ at105/pdf_files/graphs-G-LP-FPS-2015.pdf 


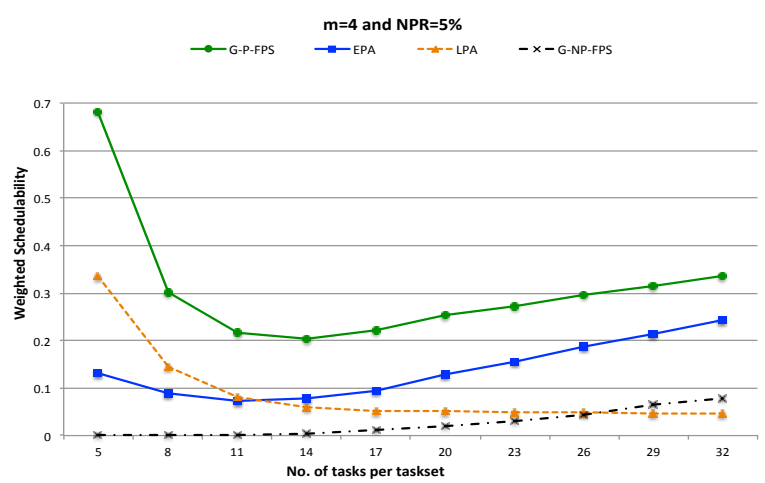

Figure 6: Varying number of tasks.

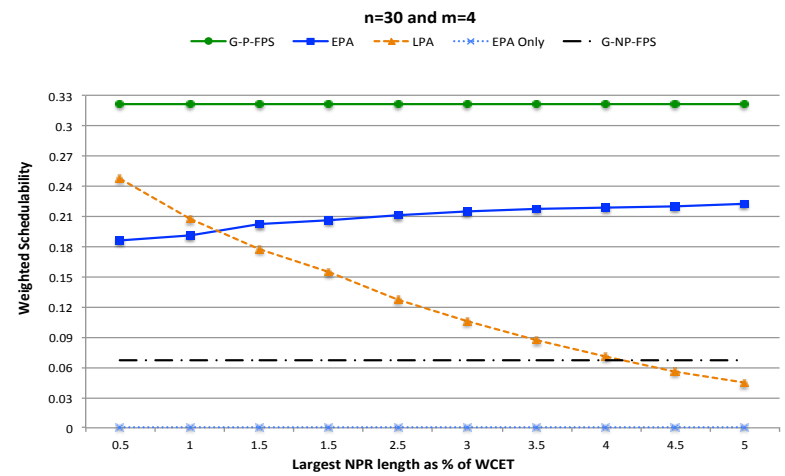

Figure 8: Varying NPR lengths (small NPRs).

schedulability. On further increasing the largest NPR lengths to $35 \%$, the number of preemption points decreases to 2 , consequently improving schedulability due to reduction of lower priority interference after the start time of the tasks. The same reasoning explains the decrease in schedulability when increasing the largest NPR lengths from $35 \%$ to $45 \%$ since the number of preemptions remain unchanged at 2. When the NPR lengths increase to $50 \%$, schedulability increases because the number of preemptions decrease to 1 . In a few cases, our experiment identified some tasksets as schedulable using G-LP-FPS with eager preemptions but unschedulable under any other approach. However, this was not observed with link-based scheduling since it is based on the test for G-P-FPS 17 after inflating the WCETs. Link based scheduling fared better compared to G-LP-FPS with eager preemptions for very small NPR lengths as shown in Figure 8.

Finally, we varied the number of processors keeping the number of tasks and NPR lengths constant, at 30 and $5 \%$ respectively (reported in Figure 9). We observe that link-based scheduling has a higher schedulability when the number of processors increases. This is due to the fact that availability of more processors implies better schedulability under link-based scheduling for low utilization tasksets with a fixed number of tasks. The same trend is seen in the left end of Figure 6 where the number of tasks compared to the available number of processors is small. However, for tasksets with a large number of tasks and high utilizations, the eager preemption approach is the

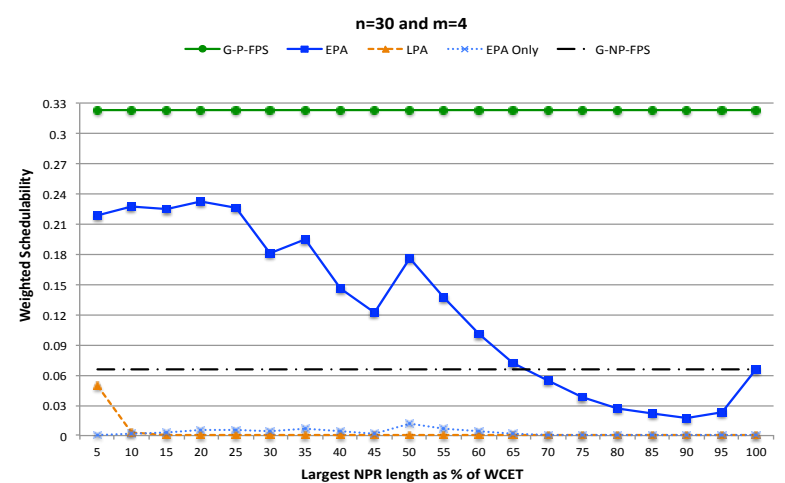

Figure 7: Varying NPR lengths (large NPRs).

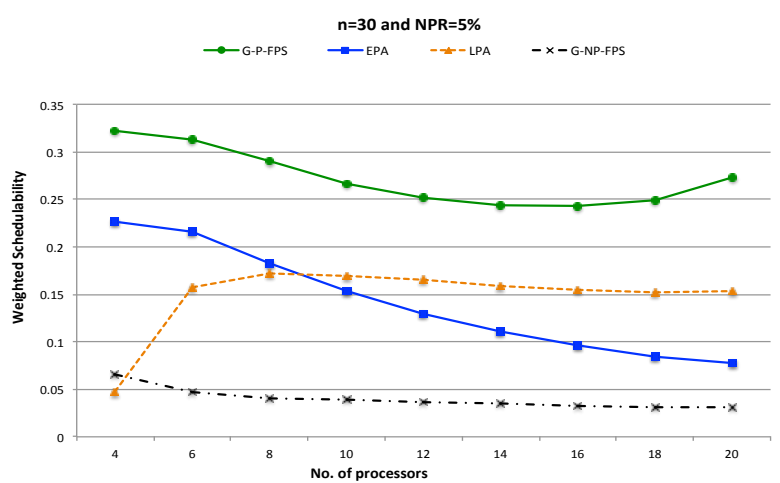

Figure 9: Varying number of processors.

most effective (Figure 6).

Even though the focus of the experiments presented in this paper is on schedulability, we note that, preliminary experiments simulating G-LP-FPS with eager and lazy preemptions suggest that the number of preemptions observed during run-time is slightly higher in the case of eager preemptions. This happens because with lazy preemptions, further higher priority tasks may be released before preemption can occur, thus the tasks are more likely to run in priority order with fewer preemptions.

\section{CONCLUSIONS}

Limiting preemptions to predetermined points within real-time tasks is an effective means of reducing preemption and migration related overheads. However, it introduces an interesting question of how best to manage preemption. At one extreme, the scheduler can choose eager preemption of the first executing lower priority task that becomes preemptable, while at the other extreme, it can restrict preemption to only the lowest priority executing task, when it becomes preemptable, referred to as lazy preemption. Each strategy has a different effect in terms of the number of priority inversions in the schedule, that in turn affects schedulability.

In this paper, we made the following contributions:

1. We derived a schedulability analysis for G-LP-FPS with eager preemptions building on the observation that blocking happens only when a task resumes 
execution. To the best our knowledge, this is the first such test for G-LP-FPS with fixed preemption points.

2. We evaluated the new schedulability test by comparing it with a test for G-LP-FPS with lazy preemption. The test used was the state-of-the-art test for G-P-FPS 17 supplemented by the inflation-based approach of accounting for blocking. 7. Our evaluations showed that G-LP-FPS with eager preemptions outperforms link-based scheduling in the context of fixed preemption points.

It remains to be seen whether the eager preemption approach is beneficial in the context of floating NPRs. Future work includes investigating optimal preemption point placement strategies, as well as experiments considering overheads on real platforms.

\section{Acknowledgments}

We would like to thank Björn Brandenburg for his insightful comments on the paper. This work was funded in part by the EPSRC project MCC (EP/K011626/1), and by the European Union, under the Seventh Framework Programme (FP7/2007-2013), grant agreement no 611016 (P-SOCRATES). EPSRC Research Data Management: No new primary data was created during this study.

\section{REFERENCES}

[1] S. Baruah. Techniques for multiprocessor global schedulability analysis. In The 28th IEEE International Real-Time Systems Symposium, 2007.

[2] A. Bastoni, B. B. Brandenburg, and J. H. Anderson. Cache-related preemption and migration delays: Empirical approximation and impact on schedulability. In The International Workshop OSPERT, 2010.

[3] M. Bertogna. Real-time scheduling analysis for multiprocessor platforms. In PhD Thesis, SSSUP, Pisa, May 2008.

[4] M. Bertogna, G. Buttazzo, M. Marinoni, G. Yao, F. Esposito, and M. Caccamo. Preemption points placement for sporadic task sets. In The Euromicro Conference on Real-Time Systems, 2010.

[5] M. Bertogna, M. Cirinei, and G. Lipari. New schedulability tests for real-time task sets scheduled by deadline monotonic on multiprocessors. In Principles of Distributed Systems, Lecture Notes in Computer Science. 2006.

[6] M. Bertogna, O. Xhani, M. Marinoni, F. Esposito, and G. Buttazzo. Optimal selection of preemption points to minimize preemption overhead. In The 23rd Euromicro Conference on Real-Time Systems, 2011.

[7] A. Block, H. Leontyev, B. Brandenburg, and J. Anderson. A flexible real-time locking protocol for multiprocessors. In The 13th IEEE International Conference on Embedded and Real-Time Computing Systems and Applications, 2007.

[8] B. Brandenburg and J. Anderson. A clarification of link-based global scheduling. In Technical Report MPI-SWS-2014-007, 2014.

[9] B. B. Brandenburg. Scheduling and Locking in Multiprocessor Real-Time Operating Systems. PhD thesis, UNC Chapel Hill, 2011.
[10] G. Buttazzo, M. Bertogna, and G. Yao. Limited preemptive scheduling for real-time systems: A survey. The IEEE Transactions on Industrial Informatics, 2012.

[11] B. Chattopadhyay and S. Baruah. Limited-preemption scheduling on multiprocessors. In Proceedings of the 22nd International Conference on Real-Time Networks and Systems. ACM, 2014.

[12] R. Davis and M. Bertogna. Optimal fixed priority scheduling with deferred pre-emption. In The Real-Time Systems Symposium, 2012.

[13] R. Davis, A. Burns, R. Bril, and J. Lukkien. Controller area network (can) schedulability analysis: Refuted, revisited and revised. Real-Time Systems, 2007.

[14] R. Davis, A. Burns, J. Marinho, V. Nelis, S. Petters, and M. Bertogna. Global fixed priority scheduling with deferred pre-emption. In The International Conference on Embedded and Real-Time Computing Systems and Applications, 2013.

[15] R. I. Davis and A. Burns. Improved priority assignment for global fixed priority pre-emptive scheduling in multiprocessor real-time systems. Real-Time Systems, 2011.

[16] R. I. Davis, A. Burns, J. Marinho, V. Nelis, S. M. Petters, and M. Bertogna. Global and partitioned multiprocessor fixed priority scheduling with deferred preemption. ACM Transactions on Embedded Computing Systems, 2015.

[17] N. Guan, M. Stigge, W. Yi, and G. Yu. New response time bounds for fixed priority multiprocessor scheduling. In The 30th IEEE Real-Time Systems Symposium, 2009.

[18] N. Guan, W. Yi, Z. Gu, and G. Yu. New schedulability test conditions for non-preemptive scheduling on multiprocessor platforms. In The IEEE International Real-time Systems Symposium, 2008.

[19] J. Y.-T. Leung and J. Whitehead. On the complexity of fixed-priority scheduling of periodic, real-time tasks. Performance Evaluation, 1982.

[20] J. Marinho, V. Nelis, S. Petters, M. Bertogna, and R. Davis. Limited pre-emptive global fixed task priority. In The International Real-time Systems Symposium, 2013.

[21] B. Peng, N. Fisher, and M. Bertogna. Explicit preemption placement for real-timeconditional code. In The Euromicro Conference on Real-Time Systems, July 2014.

[22] C. A. Phillips, C. Stein, E. Torng, and J. Wein. Optimal time-critical scheduling via resource augmentation (extended abstract). In The ACM symposium on Theory of computing, 1997.

[23] Y. Sun, G. Lipari, N. Guan, and W. Yi. Improving the response time analysis of global fixed-priority multiprocessor scheduling. In The 20th International Conference on Embedded and Real-Time Computing Systems and Applications, 2014.

[24] A. Thekkilakattil, S. Baruah, R. Dobrin, and S. Punnekkat. The global limited preemptive earliest deadline first feasibility of sporadic real-time tasks. In The 26th Euromicro Conference on Real-Time Systems, July 2014. 